\title{
Study on Threshold Behavior of Operation Voltage in Metal filament based Polymer Memory
}

Won-Jae Joo*, Tae-Lim Choi, Kwang-Hee Lee and Youngsu Chung

\section{Supplementary supporting information}

\section{TOF-SIMS analysis}

TOF-SIMS analysis was carried out to study the density of copper ions through the P3HT layer before and after the positive voltage application over the threshold one. In this measurement, the devices were newly fabricated with the structure of $\mathrm{Al}(20 \mathrm{~nm}) / \mathrm{P} 3 \mathrm{HT}(60 \mathrm{~nm}) / \mathrm{Cu}(80 \mathrm{~nm}) / \mathrm{SiO} 2(200 \mathrm{~nm}) / \mathrm{Si}$ wafer to avoid the ion beam mixing effect on copper profiles during the SIMS measurement. Three samples were prepared with different voltage bias conditions; the first device was virgin and the second and the third ones were biased for $100 \mathrm{~s}$ with lower and higher voltages (5 and $13 \mathrm{~V}$, respectively) than the threshold value (ca. $11 \mathrm{~V}$ for the device with $60 \mathrm{~nm}$ thick P3HT layer).

The voltage application was carried out in nitrogen to minimize the degradation of the devices caused by the oxygen and the moisture. There was no observable degradation in the test devices after the voltage bias.

Figure S1 shows the depth profiles of copper $\left({ }^{63} \mathrm{Cu}\right)$ and carbon $\left({ }^{12} \mathrm{C}\right)$ for the three samples above mentioned. 


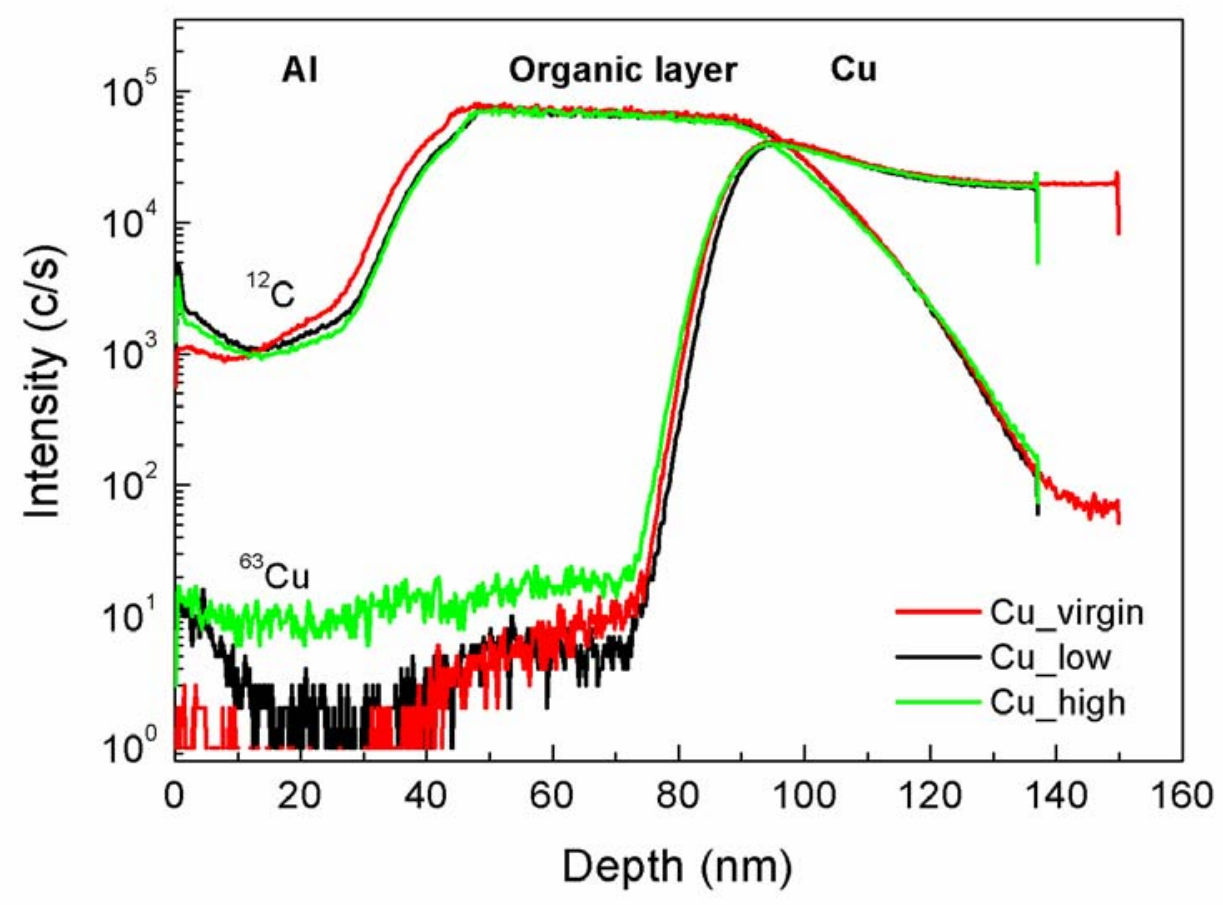

Figure S1

\section{Determination of the parameter $T_{s}$ with temperature}

A new parameter, $T_{s}$, was defined as the time when the switching probability approached to $80 \%$ in pulse voltage mode. On the other word, $T_{s}$ became the minimum time required for reproducible formation of metal filament. It was believed that $T_{s}$ was governed by the rate of electromigration of metal ions via ionization and drift processes.

The ionization and drift of copper ions were controlled by the positive voltage application. Experimentally, after applying a positive voltage (6.5 V) to the P3HT device for a relatively long time (5 s), one tested whether filament formation occurred by negative short pulse ( $-2 \mathrm{~V}$ for $0.5 \mathrm{~s}$ ) or not. Figure S2 shows the voltage pulses 
continuously applied to the device and the measured current at $40{ }^{\circ} \mathrm{C}$. The sharp peaks were observed in the current, which were caused by the formation and the cleavage of metal filament. Initially, the peaks were occasionally observed which might be due to the defects of polymer layer. However the peaks gradually increased with the positive voltage application time. After the voltage pulse bias for $460 \mathrm{~s}$, the switching probability over $80 \%$ was achieved, resulting in $T_{s}$ of $460 \mathrm{~s}$ at $40{ }^{\circ} \mathrm{C} . T_{s}$ was determined at various temperatures using this method.

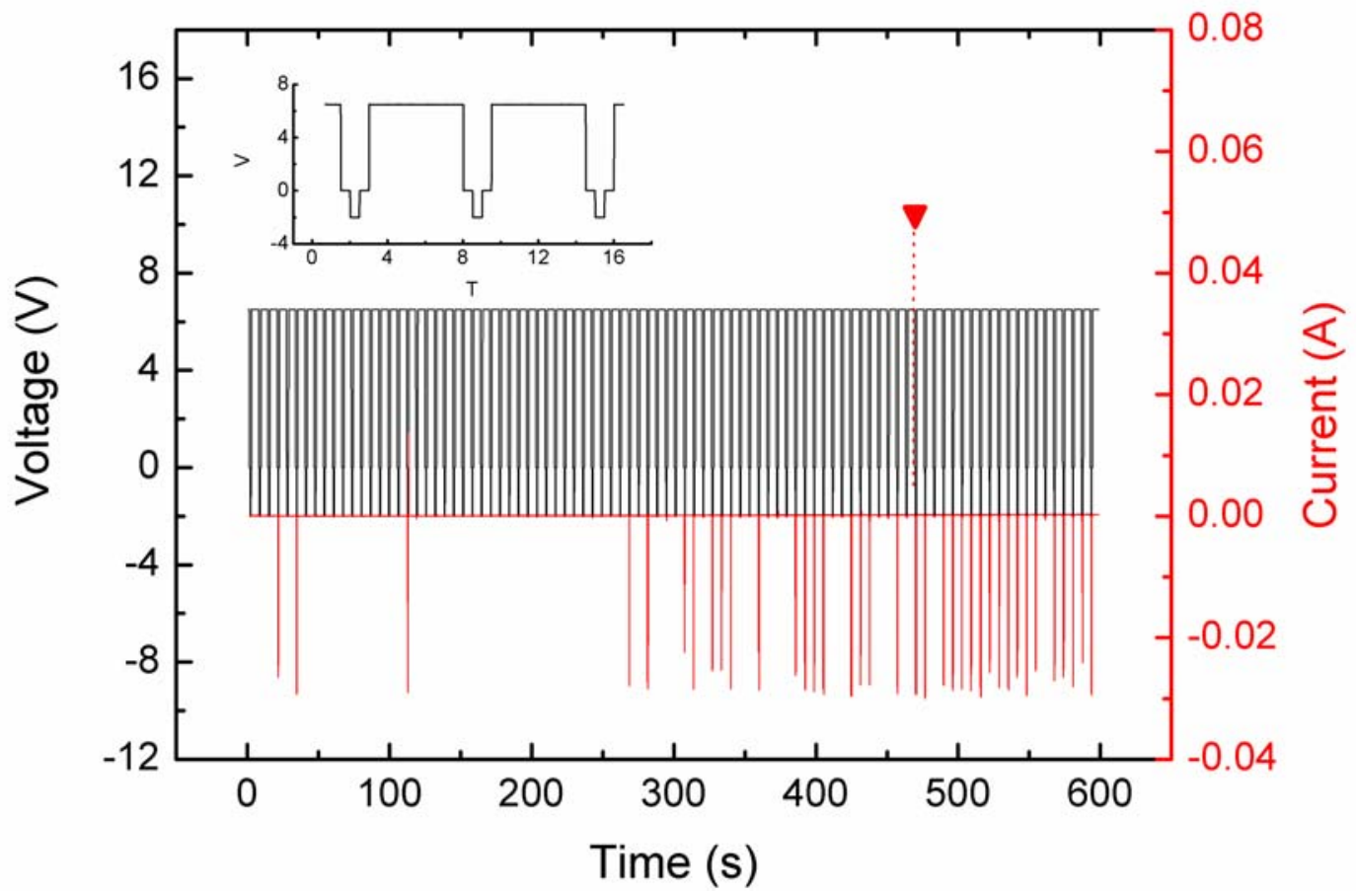

Figure S2

Because of the occasional formation of the metal filament in early stage, the waiting for $180 \mathrm{~s}$ before the actual measurement is necessary to determine the switching probability in Fig. 3. 


\section{Determination of the parameter $\boldsymbol{T}_{f}$ with electric field strength}

A new parameter, $T_{f}$, was defined as the time when the set state could not be switched back to the reset state any more by the voltage application. This failure would be due to the excess copper ions existing within polymer layer which could lead to the formation of thick metal filament or plural current paths between two electrodes. It is expected that $T_{f}$ is closely related with the switching endurance.

Experimentally, a positive voltage pulse ( $7.5 \mathrm{~V}$ for $20 \mathrm{~s}$ ) and a negative pulse (-2 V for 2 s) were continuously applied to the P3HT device (50 nm thick P3HT layer). Figure S3 shows the voltage pulses applied to the device and the current measured at room temperature. The sharp peaks were observed in the current, which were caused by the formation and the cleavage of metal filament. The switching was reproducibly observed from the initial stage of the voltage application. However, the set state was not switched back to the reset state any more by the voltage application after $630 \mathrm{~s}$. With this procedure, $T_{f}$ was determined at different electric field strength.

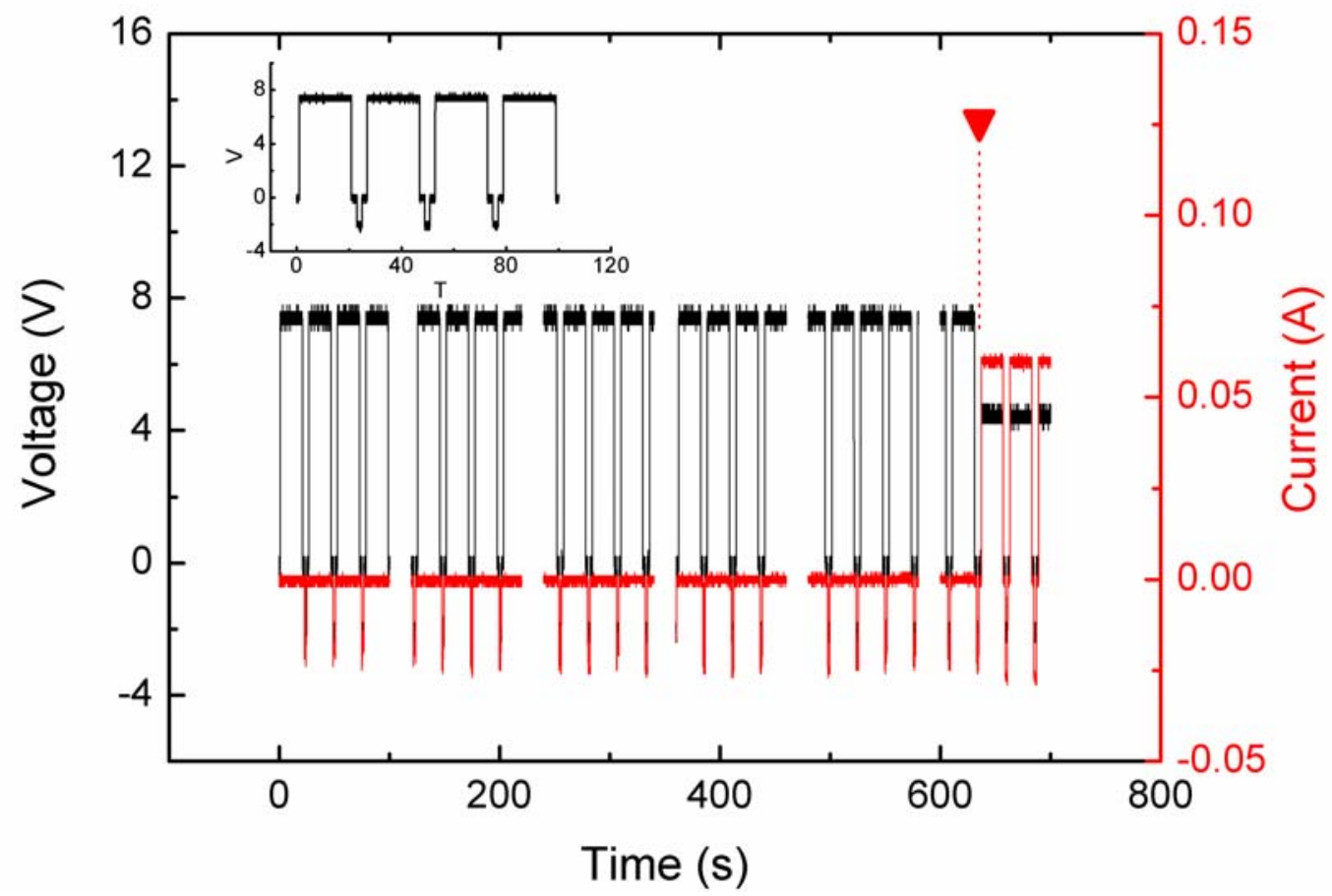

Figure S3 


\section{Switching behavior in $\mathrm{Cu} / \mathrm{P} 3 \mathrm{HT} / \mathrm{Al}$ structure}

In the $\mathrm{Cu} / \mathrm{P} 3 \mathrm{HT} / \mathrm{Al}$ structure, the similar switching behavior was observed but the switching reliability was significantly reduced. It can be caused by the absence of the aluminum oxide. The phenomenon that the switching reliability dominantly depends on the surface treatment (e.g. $\mathrm{O}_{2}$ plasma treatment) of aluminum bottom electrode was already reported in other paper.[7] Therefore, the combination of the aluminum oxide and organic material is crucial factor for the memory performance. Detailed investigation on the oxide effect should be further studied.

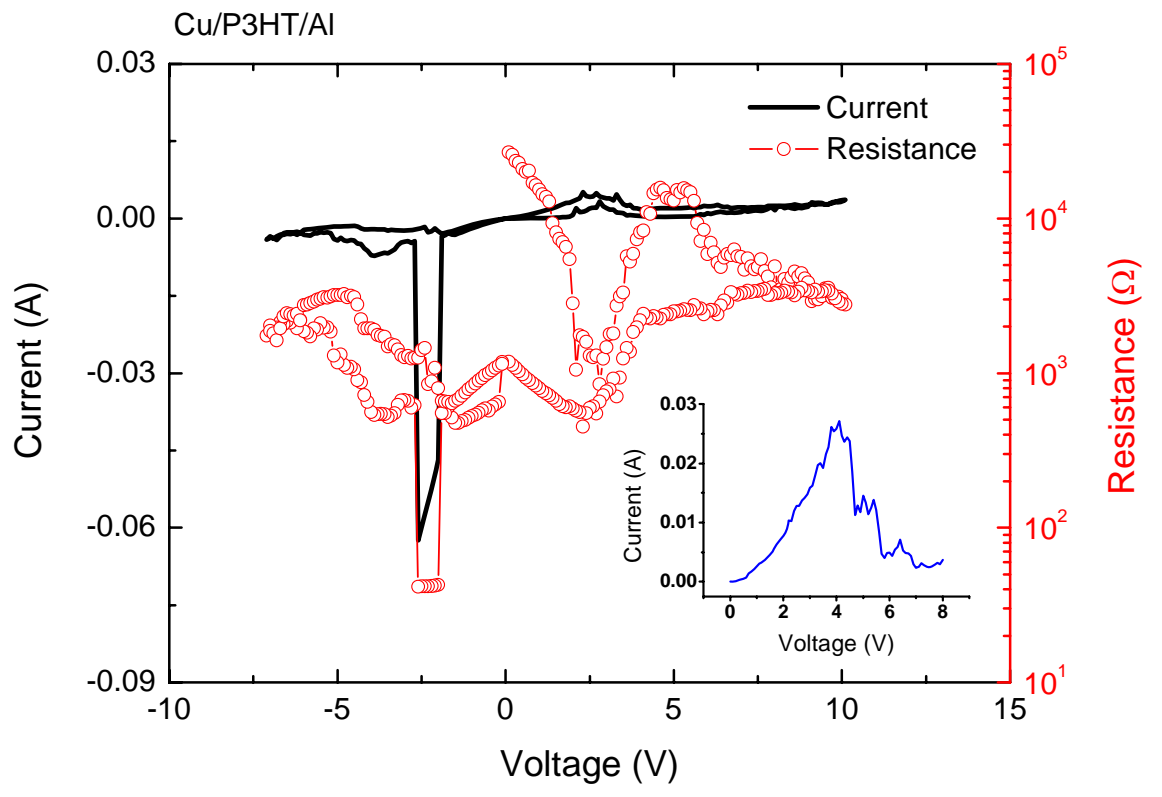

Figure S4 $\mathrm{Cu} / \mathrm{P} 3 \mathrm{HT} / \mathrm{Al}$ structure 